\title{
Electric-field dissociation of weakly bound molecular ions
}

\author{
Jesús Pérez-Ríos (1) \\ Fritz-Haber-Institut der Max-Planck-Gesellschaft, Faradayweg 4-6, D-14195 Berlin, Germany \\ and Institute for Molecules and Materials, Radboud University, Heyendaalseweg 135, 6525 AJ Nijmegen, The Netherlands
}

(Received 18 May 2021; revised 30 August 2021; accepted 31 August 2021; published 13 September 2021)

\begin{abstract}
We present a study on the dissociation of a weakly bound molecular ion in the presence of an external time-dependent electric field based on a full quantum treatment of the dynamics. We focus on the dissociation dynamics of a molecular ion in a Paul trap relevant for atom-ion hybrid traps. Our results show that a weakly bound molecular ion survives in a Paul trap giving a theoretical ground to previous experimental findings [A. Krükow et al. Phys. Rev. Lett. 116, 193201 (2016) and A. Mohammadi et al., Phys. Rev. Research 3, 013196 (2021)]. In particular, we find that weakly bound molecular ions are more likely to survive in traps with a large rf frequency. Similarly, we show that applying an electric field ramp is an efficient method to state-selectively detect weakly bound molecular ions, analogous to the well-known selective field ionization technique applied in Rydberg atoms.
\end{abstract}

DOI: 10.1103/PhysRevA.104.L031302

The advent of hybrid neutral-ion traps has boosted cold chemistry research due to the possibility of bringing together ions and atoms in a controlled manner [1-5]. Similarly, these traps find applications in different research areas such as the development of new and more efficient quantum information protocols [6-13], the realization of quantum logic spectroscopy schemes [14-22], and the study of impurity physics [23-31], to cite a few. On the impurity physics front, when a single charged impurity, $\mathrm{A}^{+}$, is brought in contact with an ultracold atomic gas $\mathrm{B}$, at sufficient densities, the ion undergoes a three-body recombination reaction: $\mathrm{A}^{+}+\mathrm{B}+\mathrm{B} \rightarrow$ $\mathrm{AB}^{+}+\mathrm{B}$ leading to the formation of weakly bound molecular ions $[1,32,33]$. In principle, these molecular ions would be hard to observe since the oscillating electric field of a Paul trap could tear them apart, or the presence of external laser sources could photodissociate them [34]. However, these weakly bound molecular ions are experimentally observed, and with it, the validity and accuracy of the classical trajectory method to calculate the three-body recombination rate is corroborated [29,32,34]. After their formation, these molecular ions relax very efficiently into deeper bound vibrational states via collisions with ultracold atoms [35,36] opening up photodissociation routes through the external laser sources characteristic to atom-ion hybrid traps [34,37].

Similarly, it has been recently predicted that a single ion in an ultracold molecular bath reacts and forms weakly bound molecular ions [38], and the same seems to occur in the case of a microwave ion clock when a single ion reacts with background gases [39-41]. In the case of a single ion in a

Published by the American Physical Society under the terms of the Creative Commons Attribution 4.0 International license. Further distribution of this work must maintain attribution to the author(s) and the published article's title, journal citation, and DOI. Open access publication funded by the Max Planck Society. molecular bath the simulations indicate that molecular ions survive despite the time-dependent electric field of the Paul trap [38]. However, the complex reaction network of a single ion in an atomic or molecular bath and the need of a quantal treatment may raise legit questions about the net effect of the time-varying electric fields on the molecular ions. Indeed, there has been little theoretical effort towards understanding the dynamics of a weakly bound molecular ion in the presence of an electric field, barring a static or effective model approach [29].

In this Letter, we present a full quantum study of the dynamics of a weakly bound molecular ion in the presence of a time-dependent electric field as well as its dissociation. In particular, we mimic the electric field that an ion feels in a Paul trap characteristic of atom-ion hybrid traps, finding that the survival of a molecular ion depends on its binding energy and on the trap frequency. In addition, we consider a Gaussian-type pulse to simulate an electric field ramp meant to dissociate the molecular ion in a controllable manner. As a result, we show that it is possible to probe the vibrational states of a weakly bound molecular ion individually via the electric field ramp method in a similar fashion to the state selective field ionization method used to ionize Rydberg atoms [42-45].

The dynamics of a molecular ion in an external electric field can be described by the following Hamiltonian:

$$
H=H_{0}+H_{\text {int }}(t)
$$

where $H_{0}$ represents the unperturbed part of the rovibrational Hamiltonian of the molecular ion. Therefore, $H_{0}$ is diagonal in the basis of the vibrational $v$ and rotational $j$ states, i.e., $H_{0}|v j m\rangle=E_{v j}|v j m\rangle$, where $E_{v j}=E_{v}+B_{v} j(j+1)$, is the vibrational energy of the $v$ th vibrational state, $B_{v}$ is the rotational constant of the assumed rigid rotor in the same vibrational state, and $m$ is the projection of the rotational quantum number on the quantization axis. $E_{v}$ and $B_{v}$ are calculated assuming a given atom-ion interaction. In Eq. (1) 


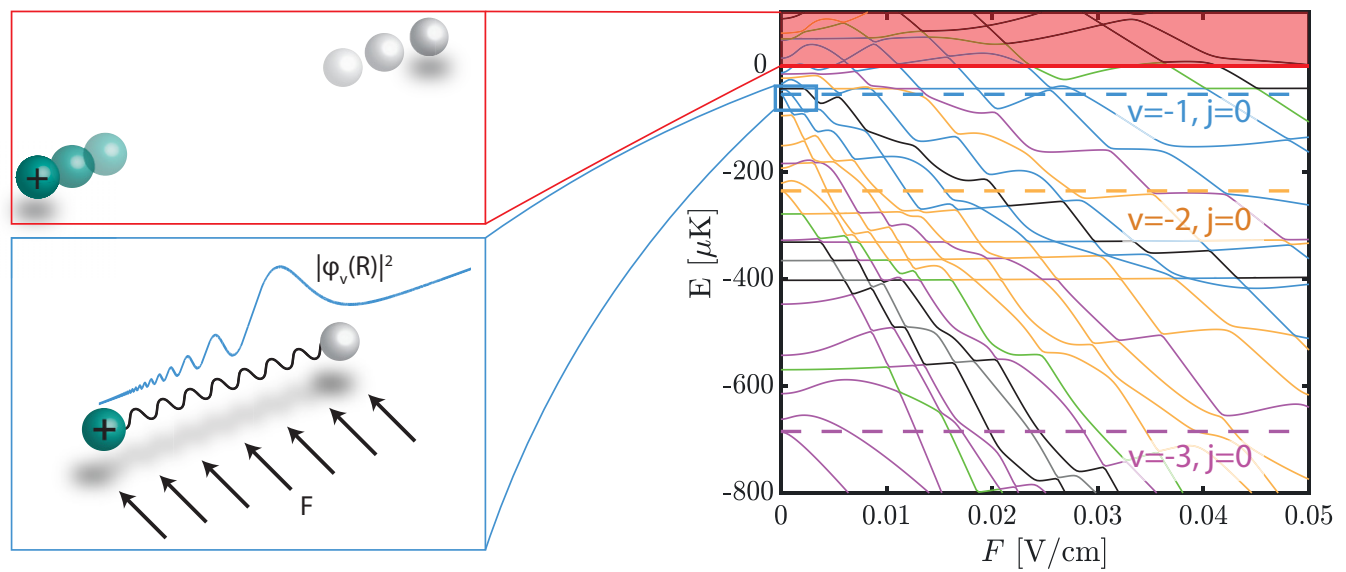

FIG. 1. Rovibrational energy spectrum of the ${ }^{174} \mathrm{Yb}^{87} \mathrm{Rb}^{+}$molecular ion as a function of the time-independent electric field strength $F$. The dashed lines correspond to a few of the relevant rovibrational states labeled by the vibrational quantum number $v$ and the rotational quantum number $j$. Lines with the same colors represent rotational states of the same vibrational state: the green lines refer to the $v=-4$, the gray line to the $v=-5$, whereas the black lines refer to deeply bound vibrational states of the molecular ion. The shaded region represents the onset of dissociation. Please note that the states above $E=0$ represent high level rotational states as a consequence of how the rovibrational energy is obtained: $E_{v j}=E_{v}+B_{v} j(j+1)$. The left panel displays schematically the nature of the molecular ion at different electric field strengths; the lower panel represents a weakly bound molecular ion, whereas the upper panel stands for a dissociated molecular ion.

the time-dependent part of the Hamiltonian is given by the interaction between the dipole moment of the molecular ion $\vec{d}$ and the time-dependent external electric field $\vec{F}(t)$ as

$$
H_{\text {int }}(t)=-\vec{d} \cdot \vec{F}(t),
$$

where we assume $\vec{d} \equiv q \vec{R}$, with $q$ denoting the charge of the ion and $R$ representing the relative position vector between the atoms within the diatomic molecular ion.

First, to illustrate the method, we calculate eigenvalues of the Hamiltonian (1) for ${ }^{174} \mathrm{Yb}^{87} \mathrm{Rb}^{+}$with $m=0$, as a function of the electric field strength, including 10 vibrational states and 20 rotational states with $j \leqslant 19$ ensuring the convergence of the results. It is worth noticing that the vibrational states are labeled from the dissociation threshold in this work, i.e., $v=$ -1 is the vibrational state with the smallest binding energy, and the larger the value of $v$, the larger the binding energy. The atom-ion interaction is taken as $-C_{4} / R^{4}\left[1-\frac{1}{2}\left(\frac{R_{m}{ }^{4}}{R}\right)\right]$ with $C_{4}=160$ a.u. and $R_{m}=10.142 a_{0}\left(a_{0} \equiv 0.529177 \times\right.$ $10^{-10} \mathrm{~m}$ ) is the equilibrium distance, corresponding to the $a^{3} \Sigma^{+}$electronic state of the system [46]. The vibrational wave functions are obtained by numerically solving the timeindependent Schrödinger equation for the vibrational motion using the Numerov method with an equispaced radial grid of $10^{5}$ steps between $5.25 a_{0}$ and $1725 a_{0} .10^{5}$ steps were used to ensure a convergence of the vibrational energies within $0.1 \%$.

In Fig. 1 we show the rovibrational energy spectrum of the least bound states of the ${ }^{174} \mathrm{Yb}^{87} \mathrm{Rb}^{+}$molecular ion as a function of the electric field strength, where it is noticed that the pathway towards dissociation (the red shaded region) is somewhat complex and highly dependent on the initial state. However, we find that $j=0$ states lower their energy as the electric field magnitude increases, whereas high $j$ states show the opposite behavior. Therefore, $j=0$ states will require a larger electric field to dissociate than states with higher $j$ values. In the same vein, focusing on the $\mid v=$ $-1, j=0\rangle$ state, we observe that it interacts with many close rovibrational states at electric fields $\lesssim 0.01 \mathrm{~V} / \mathrm{cm}$ (the same order of magnitude as the electric field that a molecular ion feels in a Paul trap), whereas higher $j$ states of the same vibrational state show a more direct pathway into the dissociation limit (the shaded red area). As a result, a static picture in which the dipole-electric field coupling lowers the dissociation threshold, leading to the dissociation of the weakly bound states, is an oversimplification of the real scenario.

To fully understand the dynamics of a weakly bound molecular ion in a time-dependent field we solve the timedependent Schrödinger equation with the Hamiltonian (1) via the ansatz

$$
|\psi(t)\rangle=\sum_{v j m} c_{v j m}(t)|v j m\rangle e^{-i E_{v j} t / \hbar},
$$

yielding the following system of coupled first-order differential equations:

$$
\begin{aligned}
\frac{d c_{v^{\prime} j^{\prime} m^{\prime}}(t)}{d t}= & -\frac{i}{\hbar} \sum_{v j m}\left\langle v^{\prime} j^{\prime} m^{\prime}\left|H_{\mathrm{int}}(t)\right| v j m\right\rangle c_{v j m}(t) \\
& \times e^{-i\left(E_{v^{\prime} j^{\prime}}-E_{v j}\right) t / \hbar},
\end{aligned}
$$

where $\hbar$ is the reduced Planck constant.

The coupling between different rovibrational states is mediated by the dipole moment of the molecular ion and reads as

$$
\begin{aligned}
& \left\langle v^{\prime} j^{\prime} m^{\prime}\left|H_{\mathrm{int}}(t)\right| v j m\right\rangle \\
& =-\left\langle v^{\prime}|d| v\right\rangle F(t) \delta_{m m^{\prime}}(-1)^{m^{\prime}} \\
& \quad \times \sqrt{(2 j+1)\left(2 j^{\prime}+1\right)}\left(\begin{array}{ccc}
j & 1 & j^{\prime} \\
m & 0 & -m
\end{array}\right)\left(\begin{array}{ccc}
j & 1 & j^{\prime} \\
0 & 0 & 0
\end{array}\right),
\end{aligned}
$$

where

$$
\left\langle v^{\prime}|d| v\right\rangle=q \int_{0}^{\infty} \phi_{v^{\prime}}^{*}(R) R \phi_{v}(R) d R
$$




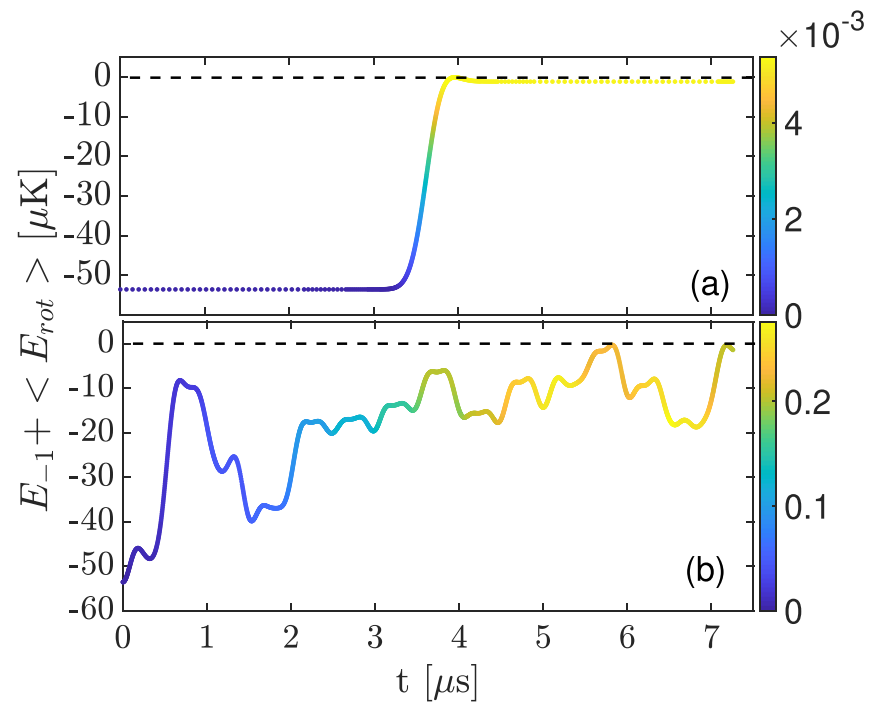

FIG. 2. Molecular ion energy of the $(v=-1 ; j=0)$ state of ${ }^{174} \mathrm{Yb}^{87} \mathrm{Rb}^{+}$as a function of time for different electric field pulses. Panel (a) is for a Gaussian pulse centered at $t_{0}=\frac{T_{\text {rot }}}{2}=\frac{\pi}{2 B_{-1}}$ with $\tau=500 \mathrm{~ns}$ and $F_{0}=0.00168 \mathrm{~V} / \mathrm{cm}$, whereas panel (b) refers to rf-type electric field with $\omega_{\mathrm{rf}}=2 \pi \times 2 \mathrm{MHz}, F_{\mathrm{dc}}=0.001 \mathrm{~V} / \mathrm{cm}$, and $F_{\text {ac }}=0.00156 \mathrm{~V} / \mathrm{cm}$. The color code refers to the probability of populating rovibrational states with $v<-1$, i.e., the vibrational quenching probability.

and (...) stands for the $3 j$ symbol. Equation (5) includes, apart from the expected rotational coupling via the $3 j$ symbols, the intravibrational coupling through the dipole moment of the molecular ion.

The time evolution of the energy associated with the $\mid v=$ $-1, j=0\rangle$ state of ${ }^{174} \mathrm{Yb}^{87} \mathrm{Rb}^{+}$for different time-dependent electric fields is shown in Fig. 2. Panel (a) displays the result for an electric field ramp to $F_{0}$ in a time $\tau$, which is simulated with a Gaussian pulse $F_{\mathrm{G}}(t)=F_{0} \exp \left[-4 \ln (2)\left(t-t_{0}\right)^{2} / \tau^{2}\right]$ centered at time $t_{0}$, with electric field strength $F_{0}$ and full width half maximum $\tau$ [47]. Whereas, panel (b) refers to an $\mathrm{rf}$ field similar to the one a molecular ion feels in a Paul trap as $F_{\mathrm{rf}}(t)=F_{\mathrm{dc}}+F_{\mathrm{ac}} \cos \left(\omega_{\mathrm{rf}} t\right)$, where $F_{\mathrm{dc}}$ and $F_{\mathrm{ac}}$ represent the $\mathrm{dc}$ and ac electric field components of the trap, respectively, and $\omega_{\mathrm{rf}}$ stands for the rf trap frequency. The energy presented is the result of adding the time-dependent rotational energy $\left\langle E_{\mathrm{rot}}\right\rangle=\sum_{v j}\left|c_{v j 0}(t)\right|^{2} B_{v} j(j+1)$ (where only $m=0$ states are considered) to the initial energy of the molecular ion's state. Indeed, as soon as this magnitude surpasses zero, the molecule dissociates. This criterion for dissociation can be viewed as a classical one since it only considers the effect of nearby vibrational states apart from the expected rotational mixing due to the electric field-dipole interaction. However, it does not include the coupling of the vibrational state of the molecular ion to the continuum. Indeed, we have estimated the contribution of continuum states to the dynamics of the relevant states to be $\lesssim 10 \%$. As a result, from Fig. 2 and as expected, the time-dependent shape of the electric field plays a major role in the dynamics of the system: for a Gaussian pulse, the molecular ion dissociates at $t \sim \tau$, whereas for the rf field, it takes a long time depending on the $\omega_{\mathrm{rf}}$. Another interesting observation from Fig. 2 is to realize that a time-dependent

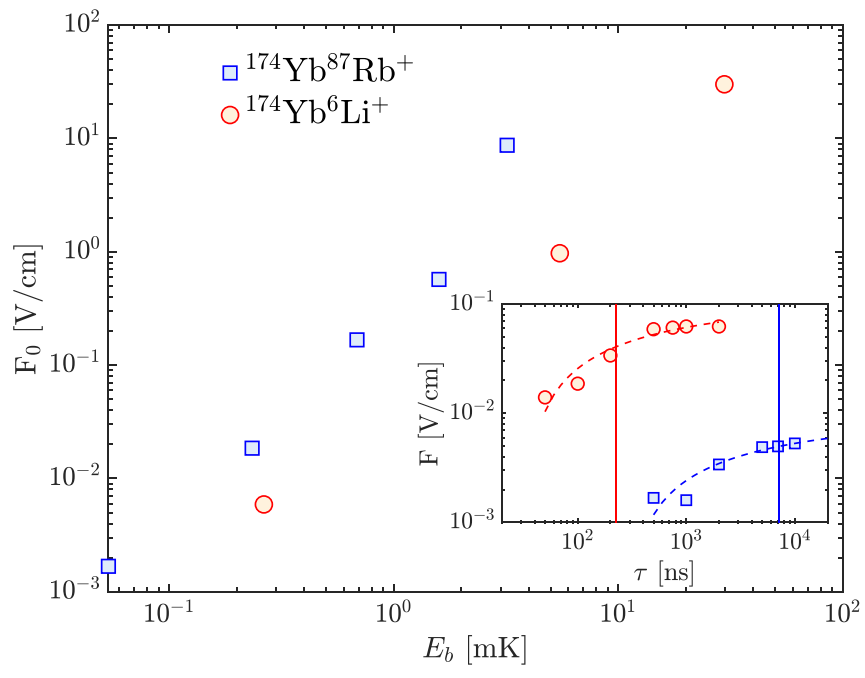

FIG. 3. Dissociation field strength for different vibrational states of ${ }^{174} \mathrm{Yb}^{87} \mathrm{Rb}^{+}$and ${ }^{174} \mathrm{Yb}^{6} \mathrm{Li}^{+}$assuming Gaussian pulse with $\tau=$ $100 \mathrm{~ns}$. The inset shows the electric field dissociation for $v=-1$ states as a function of the pulse duration $\tau$. The dashed lines correspond to a fitting of the data as explained in the text, whereas the vertical lines represent the rotational period of the involved vibrational states accordingly with the color code employed in the main figure.

electric field induces a quenching of the vibrational state of the molecular ion. In particular, the vibrational quenching probability is more pronounced in the case of an rf field than in a Gaussian pulse, implying that the dissociation pathway for a Gaussian pulse is more direct than in the case of an $\mathrm{rf}$ field.

A detailed study on the dissociation dynamics of a molecular ion due to a Gaussian pulse is shown in Fig. 3, where the electric field strength for dissociating different vibrational states with $j=0$ is displayed. The calculations for ${ }^{174} \mathrm{Yb}^{6} \mathrm{Li}^{+}$ include five vibrational states and 20 rotational states with $j \leqslant 19$ and the corresponding atom-ion interaction is characterized by $R_{m}=7.59 a_{0}$ and $C_{4}=82.1$ a.u., which correlates with its ${ }^{3} \Sigma^{+}$electronic state [48]. We notice that the larger the binding energy the larger the electric field strength needs to be, as expected. The duration of the pulse, $\tau$, affects the dynamics of the ion on the electric field and the results are shown in the inset of Fig. 3. For shorter pulses a smaller peak intensity of the electric field is required for dissociation, whereas for a duration $\tau \gtrsim T_{\text {rot }}$, where $T_{\text {rot }}=\pi / B_{-1}$ represents the rotational period (it is illustrated as the vertical solid lines), the electric field strength for dissociation tends to a constant value since the system crosses the threshold of the adiabatic limit. Indeed, we notice that the electric field strength for dissociation depends on the duration of the pulse as $F(\tau)=a+b \tau^{-1 / 4}$, and it is independent of the molecular ion species in question. For the two systems under consideration we find $a=0.009 \pm 0.001 \mathrm{~V} / \mathrm{cm}$ and $b=$ $-0.04 \pm 0.01 \mathrm{~V} / \mathrm{cm} \times \mathrm{ns}^{1 / 4}$ for the $|v=-1, j=0\rangle$ state of ${ }^{174} \mathrm{Yb}^{87} \mathrm{Rb}^{+}$, whereas $a=0.107 \pm 0.007 \mathrm{~V} / \mathrm{cm}$ and $b=$ $-0.26 \pm 0.03 \mathrm{~V} / \mathrm{cm} \times \mathrm{ns}^{1 / 4}$ for the $|v=-1, j=0\rangle$ state of ${ }^{174} \mathrm{Yb}^{6} \mathrm{Li}^{+}$. 

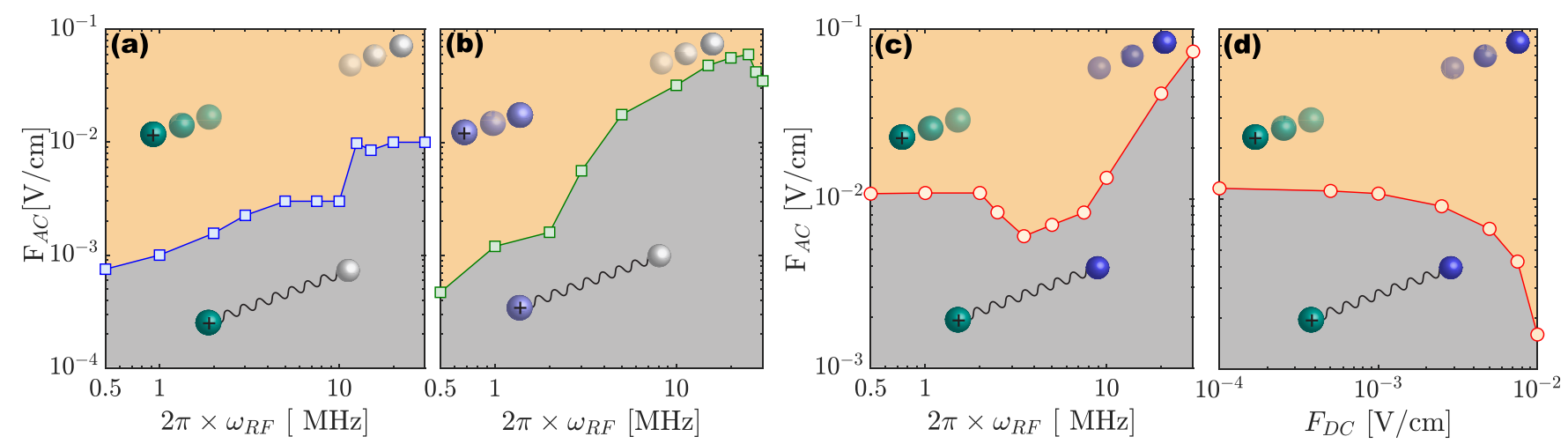

FIG. 4. Phase diagram for the dissociation of a weakly bound molecular ion. The phase diagram presents the region of the parameter space for which a given ac electric field $F_{\mathrm{ac}}$ dissociates the molecular ion for a given $\omega_{\mathrm{rf}}$ assuming a fixed dc field value of $0.001 \mathrm{~V} / \mathrm{cm}$. Panel (a) refers to the $|v=-1, j=0\rangle$ state for ${ }^{174} \mathrm{Yb}^{87} \mathrm{Rb}^{+}$. Panel (b) is for the $|v=-2, j=0\rangle$ state of ${ }^{138} \mathrm{Ba}^{87} \mathrm{Rb}^{+}$, whereas panel (c) is for the $|v=-1, j=0\rangle$ state of ${ }^{174} \mathrm{Yb}^{6} \mathrm{Li}^{+}$. In panel (d) a different phase diagram is presented for the $|v=-1, j=0\rangle$ state of ${ }^{174} \mathrm{Yb}^{6} \mathrm{Li}^{+}$, in which the ac electric field values capable to dissociate the molecular ion are shown as a function of the ac electric field for $\omega_{\mathrm{rf}}=2 \pi \times 2 \mathrm{MHz}$.

The effects of an rf-type electric field over a weakly bound molecular ion are summarized in Fig. 4, where in panels (a)-(c), the maximum amplitude of the applied ac electric field is plotted versus the rf frequency of the trap for a given value of the dc electric field. As a result, we can present a "phase space" for the dissociation of a weakly bound molecular ion in a Paul trap. The phase space shows two regions, one in which, for a given rf frequency, the molecular ion survives in a Paul trap and a second one where the molecular ion dissociates as it is schematically presented in the figure. The borderline between those regions depends profoundly on the trap frequency, the mass of the molecular ion, and its binding energy. For instance, in panels (a) and (b) the phase space for dissociation of ${ }^{174} \mathrm{Yb}^{87} \mathrm{Rb}^{+}$and ${ }^{138} \mathrm{Ba}^{87} \mathrm{Rb}^{+}$, respectively, are shown. And despite the fact that their vibrational energies are very similar - 53.6 and $81.5 \mu \mathrm{K}$ [49], respectively, the phase space looks very different. However, it is worth emphasizing that including continuum states on the dynamics will give rise to a steeper behavior of the borderline for $\mathrm{rf}$ frequencies larger than $2 \pi \times 10 \mathrm{MHz}$.

The dc electric field plays an essential role in determining the borderline between the two phases. In particular, we find that for larger dc electric fields, a smaller ac field amplitude must dissociate a molecular ion at a given frequency, as shown in panel (d) of Fig. 4, where for a fixed trap frequency it is shown the ac electric field for dissociation as a function of the dc electric field. The typical electric field strengths for dissociation range between 0.001 and $0.1 \mathrm{~V} / \mathrm{cm}$ for $\mathrm{rf}$ frequencies between $500 \mathrm{kHz}$ and $30 \mathrm{MHz}$. Nevertheless, we identify a general trend: for more significant trap frequencies, a larger ac field is necessary to dissociate the molecule. Therefore, weakly bound molecular ions are more likely to survive in high-frequency traps, independently of the mass of the constituent atoms.

The magnitude of the ac electric field that a molecular ion feels in a Paul trap depends on the position of the ion from the center of the trap, which in a cold environment depends mainly on the kinetic energy released after its formation via three-body recombination $[1,32,33,50]$ or ion-molecule collisions [33,35,38]. Indeed, the binding energy of the molecular ion is of the same order of magnitude as the collision energy [32] and in some traps, near the center of the trap, $F_{\mathrm{ac}} \sim 0.01 \mathrm{~V} / \mathrm{cm}$. Therefore, ${ }^{174} \mathrm{Yb}^{6} \mathrm{Li}^{+}$ after its formation via the reaction $\mathrm{Yb}^{+}+\mathrm{Li}_{2} \rightarrow \mathrm{YbLi}^{+}+$ $\mathrm{Li}$, survives in a Paul trap. On the contrary, ${ }^{174} \mathrm{Yb}^{87} \mathrm{Rb}^{+}$ dissociates under similar circumstances unless a large trap frequency is used. The same applies to the case of ${ }^{138} \mathrm{Ba}^{87} \mathrm{Rb}^{+}$. Finally, it is worth emphasizing that the rotational state plays a relevant role in the dissociation dynamics. In particular, rotationally excited molecular ions dissociate in the presence of smaller electric field amplitudes than in the case of $j=0$ molecular ions.

In summary, we have shown that the shape of the timedependent electric field has a drastic effect on the dissociation dynamics of a weakly bound molecular ion. As a result, after defining the phase space for molecular ion dissociation, we find that a weakly bound molecular ion is hardly dissociated in an rf trap unless minimal rf frequencies are employed, and that the required electric field depends drastically on the binding energy of the molecular ion. These results confirm previous experimental observations and reinforce theoretical predictions by including the Paul trap on the system's dynamics. Last but not the least, we have shown that an electric field ramp is an effective method to state-selectively detect molecular ions, which may have a potential impact on detecting molecular ion products of relevant cold chemical reactions such as three-body recombination and ion-molecule collisions. Finally, we would like to point out another possible mechanism for molecular ion dissociation: photodissociation. In this case, the rf trap frequency is in resonance to a continuum state, which could be studied by adapting available formalisms in the literature for Feshbach molecule association and photoionization [51-53], an idea that is currently being investigated.

The author would like to thank M. Karra and M. Mirahmadi for valuable suggestions and exciting discussions, H. Hirzler for his valuable insight regarding different 
experimental aspects of this work, M. Cretu for comments and for reading the draft and Dietrich Leibfried for useful discussion and pointing out very relevant references regarding quantum logic spectroscopy. This work has been supported by the Netherlands Organization for Scientific Research (Vrije Programma 680.92.18.05).
[1] J. Pérez-Ríos, An Introduction to Cold and Ultracold Chemistry (Springer, Cham, Switzerland, 2020).

[2] R. Côté, Chapter Two - Ultracold Hybrid Atom-Ion Systems, edited by E. Arimondo, C. C. Lin, and S. F. Yelin, Advances in Atomic, Molecular, and Optical Physics, Vol. 65 (Academic Press, New York, 2016), pp. 67-126.

[3] M. Tomza, K. Jachymski, R. Gerritsma, A. Negretti, T. Calarco, Z. Idziaszek, and P. S. Julienne, Rev. Mod. Phys. 91, 035001 (2019).

[4] P. S. Julienne, Nat. Phys. 8, 642 (2012).

[5] P. Puri, M. Mills, I. Simbotin, J. A. Montgomery, R. Côté, C. Schneider, A. G. Suits, and E. R. Hudson, Nat. Chem. 11, 615 (2019).

[6] D. Weidinger and M. Gruebele, Chem. Phys. 350, 139 (2008),

[7] K. Najafian, Z. Meir, and S. Willitsch, Phys. Chem. Chem. Phys. 22, 23083 (2020).

[8] H. Häffner, C. Roos, and R. Blatt, Phys. Rep. 469, 155 (2008).

[9] D. J. Wineland, M. Barrett, J. Britton, J. Chiaverini, B. DeMarco, W. M. Itano, B. Jelenković, C. Langer, D. Leibfried, V. Meyer, T. Rosenband, and T. Schätz, Philos. Trans. R. Soc., A 361, 1349 (2003).

[10] C. D. Bruzewicz, J. Chiaverini, R. McConnell, and J. M. Sage, Appl. Phys. Rev. 6, 021314 (2019).

[11] B. Lekitsch, S. Weidt, A. G. Fowler, K. Mølmer, S. J. Devitt, C. Wunderlich, and W. K. Hensinger, Sci. Adv. 3, e1601540 (2017),

[12] P. Schindler, D. Nigg, T. Monz, J. T. Barreiro, E. Martinez, S. X. Wang, S. Quint, M. F. Brandl, V. Nebendahl, C. F. Roos, M. Chwalla, M. Hennrich, and R. Blatt, New J. Phys. 15, 123012 (2013).

[13] J. I. Cirac and P. Zoller, Phys. Rev. Lett. 74, 4091 (1995).

[14] J. Mur-Petit, J. J. García-Ripoll, J. Pérez-Ríos, J. CamposMartínez, M. I. Hernández, and S. Willitsch, Phys. Rev. A 85, 022308 (2012).

[15] J. Mur-Petit, J. Pérez-Ríos, J. Campos-Martínez, M. I. Hernández, S. Willitsch, and J. J. García-Ripoll, in Architecture and Design of Molecule Logic Gates and Atom Circuits, edited by N. Lorente and C. Joachim (Springer, Berlin, Heidelberg, 2013), pp. 267-277.

[16] F. Wolf, Y. Wan, J. C. Heip, F. Gebert, C. Shi, and P. O. Schmidt, Nature (London) 530, 457 (2016).

[17] M. Sinhal, Z. Meir, K. Najafian, G. Hegi, and S. Willitsch, Science 367, 1213 (2020).

[18] K. Najafian, Z. Meir, M. Sinhal, and S. Willitsch, Nat. Commun. 11, 4470 (2020).

[19] A. Collopy, D. R. Leibrandt, D. G. Leibfried, and C.-W. Chou, in Optical and Quantum Sensing and Precision Metrology, edited by S. M. Shahriar and J. Scheuer, International Society for Optics and Photonics, Vol. 11700 (SPIE, Bellingham, WA, 2021).

[20] W. C. Campbell and E. R. Hudson, Phys. Rev. Lett. 125, 120501 (2020).
[21] C.-W. Chou, C. Kurz, D. B. Hume, P. N. Plessow, D. R. Leibrandt, and D. Leibfried, Nature (London) 545, 203 (2017).

[22] C. W. Chou, A. L. Collopy, C. Kurz, Y. Lin, M. E. Harding, P. N. Plessow, T. Fortier, S. Diddams, D. Leibfried, and D. R. Leibrandt, Science 367, 1458 (2020).

[23] K. S. Kleinbach, F. Engel, T. Dieterle, R. Löw, T. Pfau, and F. Meinert, Phys. Rev. Lett. 120, 193401 (2018).

[24] J. M. Schurer, P. Schmelcher, and A. Negretti, Phys. Rev. A 90, 033601 (2014).

[25] J. M. Schurer, A. Negretti, and P. Schmelcher, Phys. Rev. Lett. 119, 063001 (2017).

[26] R. Côté, V. Kharchenko, and M. D. Lukin, Phys. Rev. Lett. 89, 093001 (2002).

[27] G. E. Astrakharchik, L. A. P. Ardila, R. Schmidt, K. Jachymski, and A. Negretti, Commun. Phys. 4, 94 (2021).

[28] P. Massignan, C. J. Pethick, and H. Smith, Phys. Rev. A 71, 023606 (2005).

[29] T. Dieterle, M. Berngruber, C. Hölzl, R. Löw, K. Jachymski, T. Pfau, and F. Meinert, Phys. Rev. A 102, 041301(R) (2020).

[30] B. Midya, M. Tomza, R. Schmidt, and M. Lemeshko, Phys. Rev. A 94, 041601(R) (2016).

[31] R. Mukherjee, C. Ates, W. Li, and S. Wüster, Phys. Rev. Lett. 115, 040401 (2015).

[32] A. Krükow, A. Mohammadi, A. Härter, J. H. Denschlag, J. Pérez-Ríos, and C. H. Greene, Phys. Rev. Lett. 116, 193201 (2016).

[33] J. Pérez-Ríos, Mol. Phys. 119, e1881637 (2021).

[34] A. Mohammadi, A. Krükow, A. Mahdian, M. Deiß, J. PérezRíos, H. da Silva, M. Raoult, O. Dulieu, and J. Hecker Denschlag, Phys. Rev. Research 3, 013196 (2021).

[35] J. Pérez-Ríos, Phys. Rev. A 99, 022707 (2019).

[36] K. Jachymski and F. Meinert, Appl. Sci. 10, 2371 (2020).

[37] S. Jyothi, T. Ray, S. Dutta, A. R. Allouche, R. Vexiau, O. Dulieu, and S. A. Rangwala, Phys. Rev. Lett. 117, 213002 (2016).

[38] H. Hirzler, E. Trimby, R. S. Lous, G. C. Groenenboom, R. Gerritsma, and J. Pérez-Ríos, Phys. Rev. Research 2, 033232 (2020).

[39] T. M. Hoang, Y.-Y. Jau, R. Overstreet, and P. D. D. Schwindt, Phys. Rev. A 101, 022705 (2020).

[40] K. Sugiyama and J. Yoda, Phys. Rev. A 55, R10 (1997)).

[41] K. Sugiyama and J. Yoda, Jpn. J. Appl. Phys. 34, L584 (1995).

[42] A. M. Alonso, L. Gurung, B. A. D. Sukra, S. D. Hogan, and D. B. Cassidy, Phys. Rev. A 98, 053417 (2018).

[43] T. W. Ducas, M. G. Littman, R. R. Freeman, and D. Kleppner, Phys. Rev. Lett. 35, 366 (1975).

[44] T. F. Gallagher, L. M. Humphrey, W. E. Cooke, R. M. Hill, and S. A. Edelstein, Phys. Rev. A 16, 1098 (1977).

[45] F. Robicheaux, C. Wesdorp, and L. D. Noordam, Phys. Rev. A 62, 043404 (2000). 
[46] H. D. L. Lamb, J. F. McCann, B. M. McLaughlin, J. Goold, N. Wells, and I. Lane, Phys. Rev. A 86, 022716 (2012).

[47] M. Lemeshko and B. Friedrich, Phys. Rev. Lett. 103, 053003 (2009).

[48] M. Tomza, C. P. Koch, and R. Moszynski, Phys. Rev. A 91, 042706 (2015).

[49] The rovibrational states for ${ }^{138} \mathrm{Ba}^{87} \mathrm{Rb}^{+}$are obtained through the Numerov method using $10^{5}$ steps between $5.25 a_{0}$ and $1800 a_{0}$ and taking $R_{m}=9.27 a_{0}$ and $C_{4}=160$ a.u. for the interaction potential. The dynamics is calculated by using the 10 weakest vibrational states and including 20 rotational states $j=0-19$.

[50] J. Pérez-Ríos and C. H. Greene, J. Chem. Phys. 143, 041105 (2015).

[51] J. P. D'Incao, M. Krutzik, E. Elliott, and J. R. Williams, Phys. Rev. A 95, 012701 (2017).

[52] P. Giannakeas, L. Khaykovich, J.-M. Rost, and C. H. Greene, Phys. Rev. Lett. 123, 043204 (2019).

[53] K. Toyota, U. Saalmann, and J. M. Rost, New J. Phys. 17, 073005 (2015). 